\title{
Clade homogeneity and low rate of delta virus despite hyperendemicity of hepatitis $B$ virus in Ethiopia
}

\author{
Yeshambel Belyhun ${ }^{1,2^{*}}$, Uwe Gerd Liebert ${ }^{1}$ and Melanie Maier ${ }^{1}$
}

\begin{abstract}
Background: Although hepatitis B virus (HBV) is hyperendemic and heterogeneous in its genetic diversity in Ethiopia, little is known about hepatitis D virus (HDV) circulating genotypes and molecular diversity.

Methods: A total of 321 hepatitis B surface antigen (HBsAg) positives (125 HIV co-infected, 102 liver disease patients and 94 blood donors) were screened for anti-HDV antibody. The anti-HDV positive sera were subjected to Real time PCR for HDV-RNA confirmation. The non coding genome region (spanning from 467 to 834 nucleotides) commonly used for HDV genotyping as well as complete HDV genome were sequenced for genotyping and molecular analysis.

Results: The anti-HDV antibody was found to be 3.2\% (3) in blood donors, 8.0\% (10) in HIV co-infected individuals and $12.7 \%$ (13) in liver disease patients. None of the HIV co-infected patients who revealed HBV lamivudine (3TC) resistance at tyrosine-methionine/isoleucine-aspartate-aspartate $(\mathrm{YM}(\mathrm{I}) \mathrm{DD})$ reverse transcriptase (RT) motif with concomitant vaccine escape gene mutants was positive for anti-HDV antibody. The HDV viremia rate was $33.3 \%, 30.0 \%$ and $23.1 \%$ in respect to the above study groups. All the six isolates sequenced were phylogenetically classified as HDV genotype 1 (HDV-1) and grouped into two monophyletic clusters. Amino acid (aa) residues analysis of clathrin heavy chain (CHC) domain and the isoprenylation signal site (Py) at 19 carboxyl (C)-terminal amino acids (aa 196-214) and the HDV RNA binding domain (aa 79-107) were highly conserved and showed a very little nucleotide variations. All the sequenced isolates showed serine at amino acid position 202. The RNA editing targets of the anti-genomic HDV RNA (nt1012) and its corresponding genomic RNA (nt 580) showed nucleotides A and C, respectively.

Conclusions: The low seroprevalence and viraemic rates of HDV in particular during HIV-confection might be highly affected by HBV drug resistance selected HBsAg mutant variants in this setting, although HDV-1 sequences analysis revealed clade homogeneity and highly conserved structural and functional domains. Thus, the potential role of HBV drug resistance associated polymerase mutations and concomitant HBsAg protein variability on HDV viral assembly, secretion and infectivity needs further investigation.
\end{abstract}

Keywords: HDV, Genotype, Clade homogeneity, Ethiopia

\section{Background}

HDV is a defective RNA virus and an obligate satellite of HBV that infects humans either concomitantly with HBV or after HBV infection [1]. Its genome is a circular, negative sense, and single-stranded RNA of approximately $1.7 \mathrm{~kb}$ in length [1].The HDV anti-genome contains a unique open reading frame that encodes the

\footnotetext{
* Correspondence: belyhun@gmail.com

${ }^{1}$ Institute of Virology, Medical Faculty, Leipzig University, Johannisallee 30, 04103 Leipzig, Germany

${ }^{2}$ School of Biomedical and Laboratory Sciences, College of Medicine and Health Sciences, University of Gondar, Gondar, Ethiopia
}

small (sHDAg) and large (LHDAg) hepatitis delta antigens from the same reading frame with amino acids length of 195 and 214, respectively [1, 2]. HDV prevalence and geographic distribution is not uniform and epidemiological information from most parts of the world is still lacking [3]. However, it is generally considered to be endemic in Africa, Asia, Latin America, Mediterranean regions and Eastern Europe [3, 4]. In contrast, HDV prevalence is still low in the developed western countries $[4,5]$ despite a changing pattern due to immigration from the endemic regions $[4,5]$. From 
the globally estimated 350 million HBsAg carriers, 15 to 20 million people are infected with HDV which is associated with a severe form of viral hepatitis [6]. Although the mechanism of HDV pathogenesis has not been fully explained [3], the severity of liver disease due to HDV varies by the nature of its co-or super-infection with HBV and as well the genotypes of both viruses [7].

Clinically, the co-infection of HBV with HDV leads to acute hepatitis, but subsequent chronic infection is rare, whereas the super-infection of HDV in HBV carriers typically induces a severe form of hepatitis and causes chronic infection compared to HBV monoinfection [3]. The variation in clinical severity of HDV may also be determined by host and viral genetic factors and the geographical discrepancies by the disease severity is usually associated with genotypes of HDV [8]. To date, eight distinct HDV clades or genotypes are recognized based on their sequence homology and are labeled as HDV-1 to HDV-8 [9]. Still, to characterize more HDV isolates from different parts of the world have a crucial virological and clinical meaning [8]. In addition, whether combinations of HBV genotypes with HDV genotypes cause varying clinical outcomes, remains to be explored $[8,10]$.

Moreover, compared with HBV mono-infection, human immunodeficiency virus (HIV)-HBV co-infections may lead to accelerated hepatic disease progression with higher rates of liver cirrhosis and liver-related mortality and complicate treatment possibilities [11]. Notably, rate of HDV infection has been increasing among HBV-HIVco-infected patients [10]. Nevertheless, there is scarce information about HDV prevalence, virological profile and its natural history among HIV patients [7] especially in areas where HDV is endemic and routine HBV immunization is not common [12]. In particular, while HBV seroprevalence is very high across African countries [13], little information is available on the HDV origin, circulation, and molecular diversity. Similarly, in Ethiopia where HBV remains unchecked for clinically suspected liver disease patients and HIV co-infected individuals coupled with absence of HBV vaccination coverage for the adult population, molecular epidemiology and clinical spectrum of liver disease due to HDV viral origin are unknown. So far, there is no molecular information about HDV from Ethiopia. This study was, therefore, aimed to determine molecular epidemiology and genetic diversity of HDV isolated from HBV-HIV co-infected and HBV mono-infected Ethiopians.

\section{Methods}

\section{Patients/study participants}

In 2013, a total of 4105 study samples were collected from HIV infected individuals $(n=2133)$, blood donors $(n=1720)$ and chronic liver disease (CLD) patients $(n=252)$ in northwest Ethiopia. Among this total study population, $340 \mathrm{HBsAg}$ positive sera were studied before to determine clinically important HBV drug resistant and immune (vaccine) escape HBsAg gene variants among HIV co-infected and HBV monoinfected Ethiopians [14]. In this cross-sectional study, from the above well characterized $\mathrm{HBsAg}$ positive sera, 321 of them were screened for anti-HDV antibody with the proportions of blood donors $(n=94)$, CLD patients $(n=102)$ and HIV co-infected patients $(n=125)$. The demographic data and clinical characteristics of the study subjects screened for HDV were collected during blood sample collection as described earlier [14].

\section{HDV antibody screening}

A total of $321 \mathrm{HBsAg}$ positives sera was tested for qualitative total anti-HDV antibody (IgG-Ab) using commercially available kit ETI-AB-DELTAK-2 (DiaSorin S.P.A., Saluggia, Italy). The sera were also characterized before [14] for the status of HIV and other hepatitis viruses such as $\mathrm{HBV}$ and $\mathrm{HCV}$.

\section{HDV RNA isolation, quantification and short fragment amplification}

HDV RNA was extracted using $200 \mu \mathrm{l}$ sera from 26 anti-HDV antibody positives cases applying MagNA Pure 96 DNA and Viral NA Small Volume kit on the MagNA Pure 96 Instrument (Roche Diagnostics, Mannheim, Germany). Reverse transcription-Real-Time PCR (RT-rt. PCR) was done for the partial fragment non coding genome region with a spanning of 467-834 nucleotides that commonly used for HDV genotyping [15]. In brief, $5 \mu \mathrm{l}$ of extracted RNA was heat-incubated at $70{ }^{\circ} \mathrm{C}$ for $5 \mathrm{~min}$ to diminish secondary structures. The RNA was then reverse transcribed using Super Script III reverse transcriptase enzyme (Invitrogen, Karlsruhe, Germany) and Random-Hexamer-Primers (Roche Applied Science, Mannheim, Germany). The RT was performed in a final volume of $20 \mu \mathrm{l}$ with $4 \mu \mathrm{l}$ of $5 \times$ First strand buffer, $0.1 \mu \mathrm{l}$ of dithiothreitol $(0.1 \mathrm{~mol} / \mathrm{l})$, $0.75 \mu \mathrm{l}$ random Hexames primers $(400 \mathrm{ng} / \mu \mathrm{l}), 3 \mu \mathrm{l}$ of dNTP's $(2.5 \mathrm{mmol} / \mathrm{l})$ and $1 \mu \mathrm{l}$ RNase-inhibitor $(40 \mathrm{U} / \mu \mathrm{l})$. The final RT was performed for $5 \mathrm{~min}$ at $25^{\circ} \mathrm{C}$, followed by $50{ }^{\circ} \mathrm{C}$ for $35 \mathrm{~min}$ and heat activated for $2 \mathrm{~min}$ at $95^{\circ} \mathrm{C}$. Two microliters of cDNA were subsequently applied to Real-Time-PCR with PlatinumTaq-Polymerase (Invitrogen, Karlsruhe, Germany) on a LightCycler 2.0 instrument (Roche Diagnostics, Mannheim, Germany) in a $20 \mu \mathrm{l} \mathrm{re-}$ action mixture containing $2.0 \mu \mathrm{l}$ of $5 \times$ Buffer, $1.6 \mu \mathrm{l}$ of $\mathrm{MgCl}_{2}(50 \mathrm{mM})$ BSA $(2 \mathrm{mg} / \mathrm{ml})$, dNTP, $0.4 \mu \mathrm{l}$ of PlatinumTaq Polymerase $(5 \mathrm{U} / \mu \mathrm{l}), 10 \mu \mathrm{M}$ of primer HD10 (5'-AGT GAG GCT TAT CCC GG-3') and primer HD11 (5'-CTC GGA TGG CTA AGG GAG-3'), $12 \mu \mathrm{M}$ of 
fluorescein labelled locked nucleic acid probe HD LNA $\left(5^{\prime}-\mathrm{AG}+\mathrm{CCT}+\mathrm{CCT}+\mathrm{CG}+\right.$ CTGGCGCC-FL-3') and $4 \mu \mathrm{M}$ of modified HDV1 light cycler red (LCR) 640 probe (5' - LCRed640-GCTGGGCAACATTCCGAGG-ph-3'). The PCR temperature profiles was as follows: denaturation at $95{ }^{\circ} \mathrm{C}$ for $5 \mathrm{~min}$, followed by 15 touch-downcycles of $95^{\circ}$ denaturation for $5 \mathrm{~s}$, annealing at $62-48{ }^{\circ} \mathrm{C}$ with $1^{\circ}$ reduction each cycle, and elongation at $72{ }^{\circ} \mathrm{C}$ for $10 \mathrm{~s}$ without measurement and followed by 35 cycles with annealing at $48^{\circ}$ and measurement at $640 \mathrm{~nm}$.

\section{HDV full genome amplification and sequencing}

The complete genome amplification of six patients' HDV RNA positive samples was performed. The need for full genome characterization was necessitated since the above partial fragment (368 nt length) sequences of all the six isolates were genotyped as HDV-1. To produce two partially overlapping segments covering the entire HDV genome $(\approx 1670 \mathrm{nt})$, two sets of primers from Çelik et al. [8] were used with certain modifications. The forward (5-GCG GGC CGG CTACTC TTC TTT C-3'; nt 1160-1180) and the reverse primers (5'CTA GCC CCG TTG CTT TCT TTG CTT T-3'; nt 410-130) were used for the first fragment amplification covering $950 \mathrm{bp}$. The other set of primes (forward: $5^{\prime}-$ ACC TCCAGA GGA CCC CTT CAG CGA A-3'; nt 300-320 and reverse: 5'-GAG GGA GCT CCC CCG GCG AAGAG-3'; nt 1580-1600) were used for the second fragment amplification covering $1100 \mathrm{bp}$. The RT reaction was performed at $50{ }^{\circ} \mathrm{C}$ incubation using Super Script III reverse transcriptase enzyme (Invitrogen, Karlsruhe, Germany) for $1 \mathrm{~h}$ in a total volume of $20 \mu \mathrm{l}$ RT mixture of $5 \times$ First strand buffer, $10 \mathrm{mM}$ dNTPmix (Roche Applied Science, Mannheim, Germany), $0.1 \mathrm{M}$ DTT, $10 \mu \mathrm{M}$ reverse primers, $40 \mathrm{U} / \mu \mathrm{l}$ Fermentas RiboLock and $200 \mathrm{U} / \mu \mathrm{l}$ and $5 \mu \mathrm{l}$ template RNA. The PCR were performed with $5 \mu \mathrm{l}$ of cDNA mixed with $10 \mu \mathrm{M}$ of each primer, $10 \mathrm{mM}$ dNTPmix, $50 \mathrm{mM} \mathrm{MgCl} 2,5 \mathrm{U} /$ $\mu \mathrm{l}$ Taq DNA polymerase (Promega, Madison, WI, USA) and $10 \times$ buffer to a final volume of $50 \mu \mathrm{l}$. Then the PCR was performed at $94{ }^{\circ} \mathrm{C}$ for $1.5 \mathrm{~min}$ followed by 35 cycles at $94{ }^{\circ} \mathrm{C}$ for $30 \mathrm{~s}, 55^{\circ} \mathrm{C}$ for $30 \mathrm{~s}$ and $72{ }^{\circ} \mathrm{C}$ for $1.5 \mathrm{~min}$ and a final extension for $3 \mathrm{~min}$ at $72{ }^{\circ} \mathrm{C}$. The RT and PCR reaction components, concentrations and conditions used for both fragments were similar. The amplified PCR products for both non-coding partial genome region and the full genome were gel extracted from $1.5 \%$ agarose gel and purified using the Wizard SV Gel and PCR Clean-Up System (Promega, Mannheim, Germany). Strict precautions were followed to avoid cross contamination and appropriate positive and negative controls were included during DNA extraction and PCR amplification steps. Finally, the sequence products were subjected to direct sequencing using the ABI Prism BigDye
Terminator cycle sequencing reaction kit (Applied Biosystems, Foster City, CA, USA) using ABI Prism 3500 Genetic Analyzer.

\section{Phylogenetic and sequence analysis}

All raw sequences were manually edited and aligned using Geneious software version 6.2.1 (http://www.geneious.com). Neighbor-joining phylogenetic trees and genetic distances were calculated using MEGA5 software (www.megasoftware.net) with Kimura 2-parameter, pair wise deletion option, and 1000 bootstrap replicates. Genotyping was independently performed on the basis of phylogenetic relationship by taking the short fragment non-coding genome region $(368 \mathrm{pb})$ and the full HDV genome sequences along with representative reference sequences from different HDV genotypes of 1 to 8 retrieved from the GenBank. Alignment of both the nucleotide sequences and the amino acid residues of the current isolates that encode 19 carboxyl (C)-terminal amino acid (aa 196-214) residues of LHDAg depicting the clathrin heavy chain $(\mathrm{CHC})$ interacting domain (includes clathrin box-binding domain (CBD) (aa 199203), the isoprenylation (Py) signal site (aa 211-214)) were compared with sequences retrieved from the GenBank. Moreover, nucleotide substitutions variations on the poly(A) signal (nt 946-952), and the RNA editing targets of the anti-genomic HDV RNA (nt 1012) and its corresponding genomic RNA (nt 580) were examined for nucleotide substitution variations. The acetylation (Ac) and phosphorylation (Pi) sites, RNA binding domain (aa 79-107) and the domains of arginine-rich motifs (ARMs) (aa 137-144) of sHDAg were also analysed for possible nucleotide and amino acid residue variations. The nucleotide sequences of both non-coding short fragment genome region and the full HDV genome region used in this study are available in the GenBank/ EMBL/DDBJ data bases with an accession numbers from KY463671 to KY463682.

\section{Statistical analysis}

Statistical analysis was performed by using GraphPad Prism Software version 5.01, 2007. Non-parametric data were compared by using the Mann-Whitney $U$ test and categorical data were compared by Chi-Square test. A $P$ value of less than 0.05 considered to be statistically significant.

\section{Results}

\section{Patients/Clients}

The anti-HDV positive patients/ clients median interquartile (IQR) age was 38 (30-48) years and their demographic, clinical and virological characteristics are presented in Table 1. 


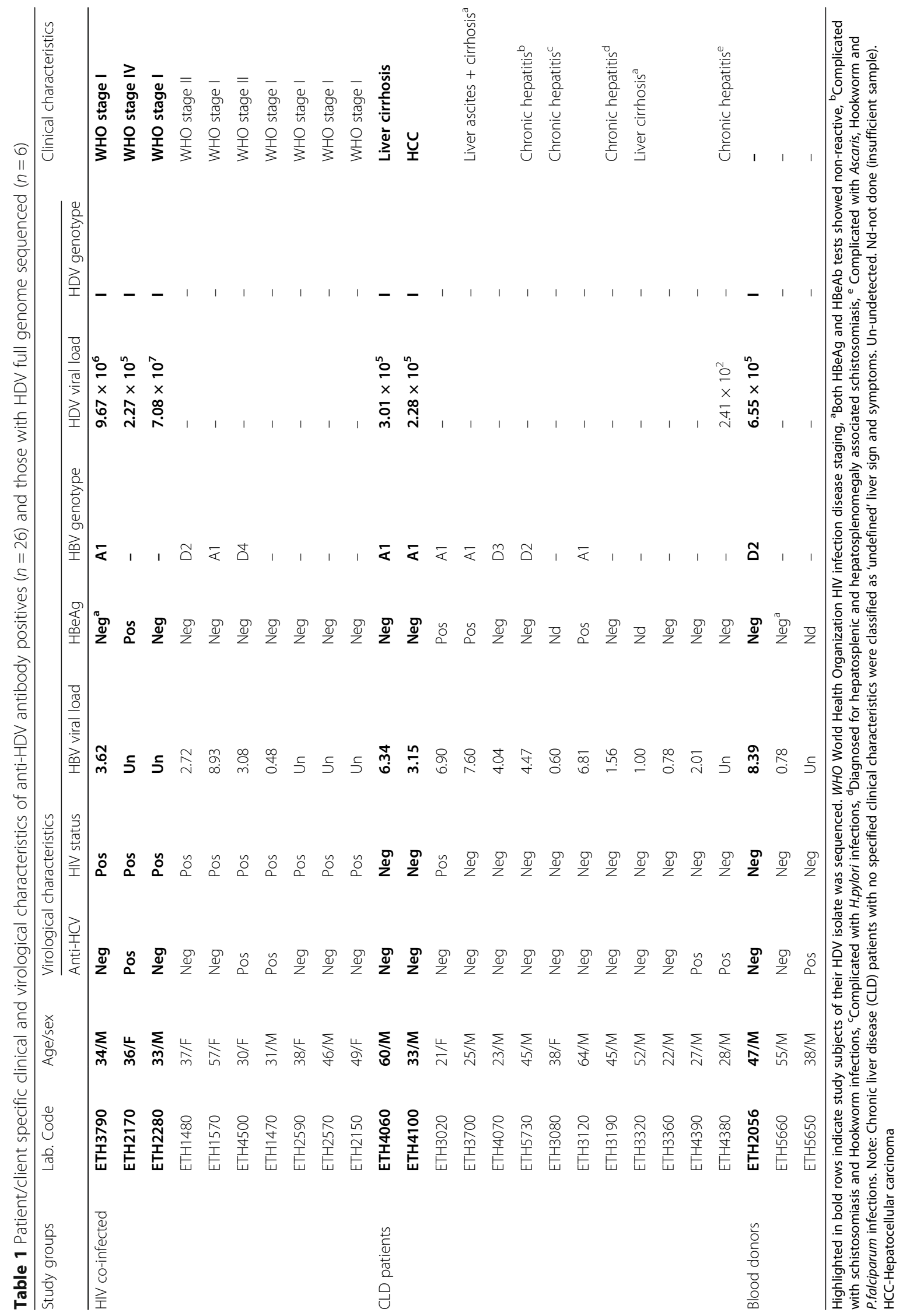


Clinically, three of the six sequenced isolates were from HIV-HBV-HDV triple co-infected patients who were on their HIV ART follow up for 2, 7.7 and 8.1 years whereby isolates ETH3790 and ETH2280 were WHO stage I and the other isolate ETH2170 was stage IV (Table 1). The two patients with isolates ETH4060 and ETH4100 were clinically diagnosed for liver diseases; isolate ETH4060 with liver cirrhosis (LC) and ETH4100 with hepatocellular carcinoma (HCC) (Table 1). The ETH20560 isolate was from an apparently healthy blood donor (Table 1).

\section{Virological characteristics}

The anti-HDV prevalence among HBsAg positive samples ranges from $3.2 \%$ (3) in blood donors, $8.0 \%$ (10) in HBV-HIV co-infected individuals to $12.7 \%$ (13) in liver disease patients. Interestingly, none of the HBV-HIV coinfected patients who were characterized by YM(I)DD motif 3TC resistance gene mutations selected vaccine escape HBsAg gene variants (sE164D/sI195M/sW196*) [14] were positive for anti-HDV antibody. The viral RNA positivity rate was $33.3 \%$ (1), $30.0 \%$ (3) and $23.1 \%$ (3) from blood donors, HBV-HIV co-infected and CLD patients, respectively (Fig. 1).

Six out of the seven sera with detectable viral load were successfully sequenced and the HDV viral load ranged from $2.27 \times 10^{5}$ to $9.67 \times 10^{6}$ copies $/ \mathrm{mL}$ (Table 1 ). The seroprevalence of HBsAg were reported before as $8.4 \%$ in blood donors, $6.7 \%$ in HIV co-infected and 43.3\% in CLD patients [14] but the actual prevalence of

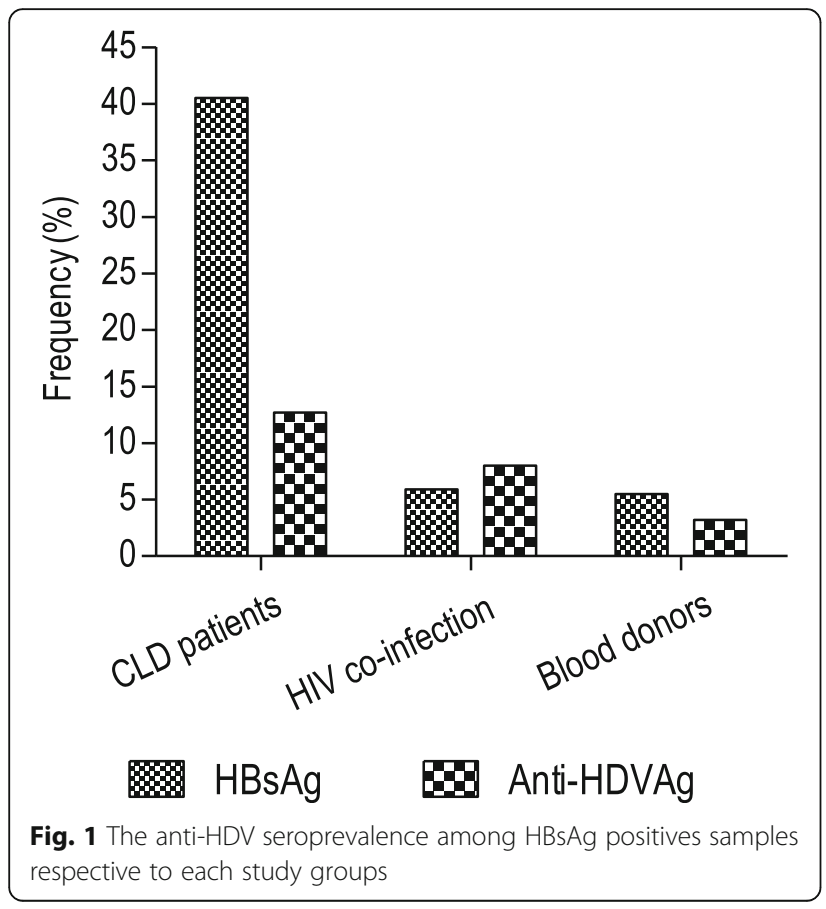

HBsAg, in which anti-HDV was currently screened, was $5.5 \%, 5.9 \%$ and $40.5 \%$ among the above respective groups (Fig. 1). The HBV DNA was detected with the median (IQR) viral load level of $3.39 \mathrm{log} \mathrm{IU} / \mathrm{mL}(1.42-$ 6.83) from $69.2 \%$ (18) anti-HDV positive sera samples. From $30.8 \%$ (8) anti-HDV positive cases, HBV DNA was undetected (Table 1). Among the six study subjects with HDV RNA sequenced, isolates ETH2170 and ETH2280 were included in the latter group but isolates; ETH3790, ETH4060, ETH4100 and ETH2056 showed HBV DNA viral load of $3.62 \log \mathrm{IU} / \mathrm{mL}, 6.34 \log \mathrm{IU} / \mathrm{Ml}, 3.15 \log$ $\mathrm{IU} / \mathrm{mL}$ and $8.39 \log \mathrm{IU} / \mathrm{mL}$, respectively (Table 1). Except isolate ETH2056 which was subgenotype D2, three of the above isolates were HBV subgenotype A1. All of the study subjects who were screened for anti-HDV antibody were characterized before for their anti-HCV status. However, none of the anti-HDV antibody positive study subjects were HCV PCR positive (Table 1).

\section{Phylogenetic analysis}

The phylogenetic analysis from both non-coding partial genome region (368 nt) and the full genome sequences ( 1678 nt) classified all the six isolates as HDV genotype 1 (HDV-1) (Fig. 2). The analysis of the sequences from the current study and reference sequences retrieved from the GenBank revealed that the current HDV-1 strains formed two monophyletic clusters in the phylogenetic analysis. The isolates ETH2170, ETH2056, ETH3790 and ETH4100 formed one independent cluster together with previously described single Ethiopian strain particularly in the full genome sequences analysis (Fig. 2b). The other two strains; ETH2280 and ETH4060 mostly clustered with West African strains (Fig. 2). Interestingly, this independent clustering was reproducible during the phylogenetic analysis of the full genome as well as the non-coding partial genome region sequences (Fig. 2a and b).

\section{Amino acid residues and nucleotides analysis}

The CHC interacting domain at 19 C-terminal $\left({ }^{196}\right.$ WDILFPSDPPFSPQS-CRPQ $\left.{ }^{214}\right)$ showed no amino acid variations in all of the six isolates. This includes the CBD $\left({ }^{199}\right.$ LFPSD $\left.^{203}\right)$ and the isoprenylation (Py) signal site $\left({ }^{(211} \mathrm{CRPQ}^{214}\right)$ (Fig. 3). All of the six isolates also showed serine (S) at amino acid position 202. The homology at nucleotide level in both CBD and Py regions were also compared with HDV-1 sequences retrieved from GenBank and showed high conservation with no nucleotide variations (Fig. 4a). Similarly, all of the isolates showed no nucleotide variations of the poly(A) signial domain sequences (946-CTTTATT-952) when compared to the refrecne strains of genotypes $1-3$. The 


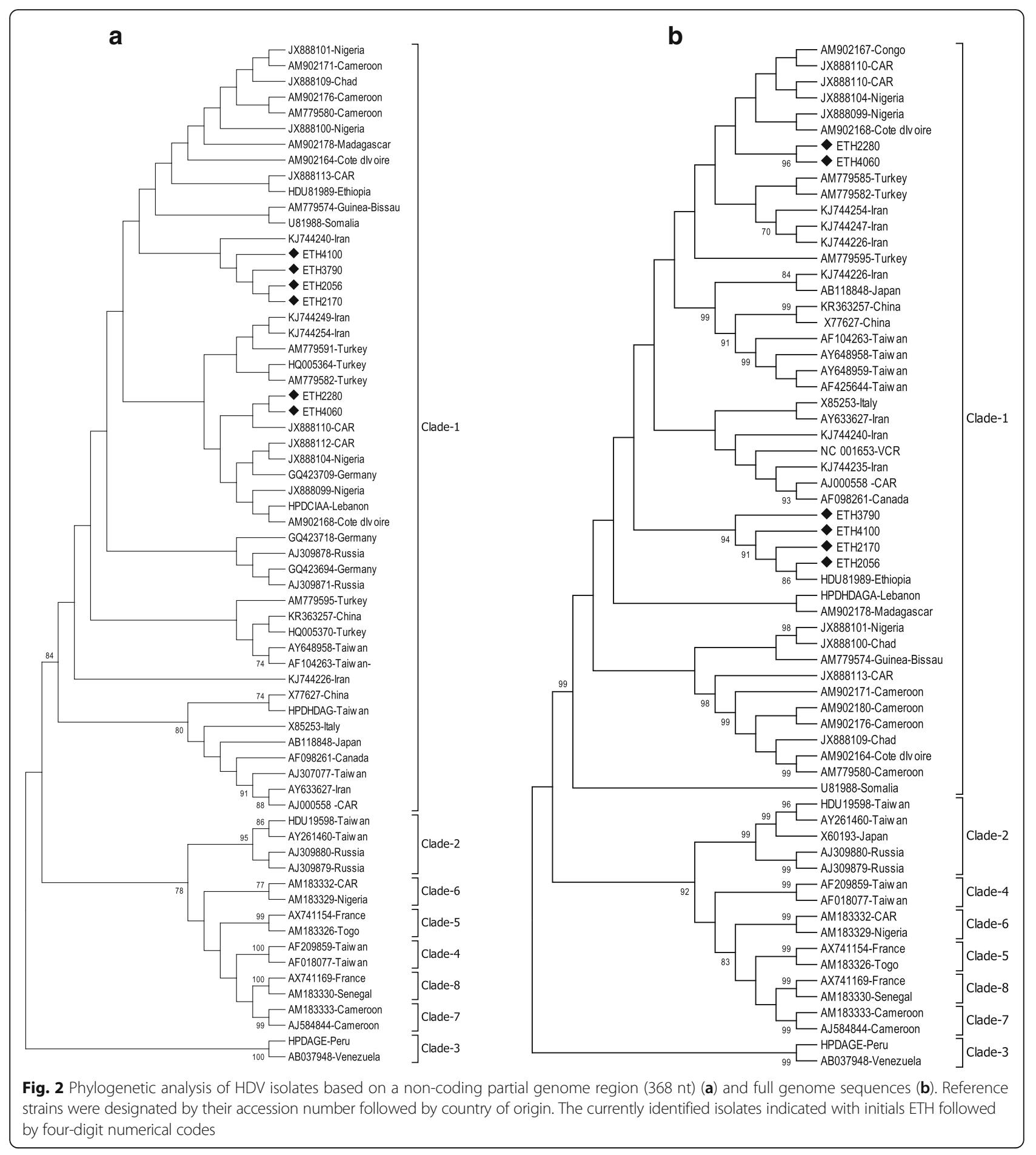

RNA editing targets of the anti-genomic HDV RNA (at nt 1012) and its corresponding genomic RNA (at nt 580) showed nucleotide A and C, respectively. However, unusual nucleotide changes (A to $G$ ) at nt 1014 were observed among four of the isolates (Fig. 4b). In the HDV-1 RNA editing domain of the sHDAg (aa 79-107), all of the six isolates showed amino acid changes only at two sites (aa 80 and 88) (Fig. 3). Moreover, the acetylation (AC) site (aa 72) revealed a lysine residue in all of the isolates. Similarly the amino acid compositions of the phosphorylation ( $\mathrm{Pi}$ ) sites (aa 2, 123, and 177) showed a serine residue in all the isolates. The domains of ARMs (aa 137-144) relatively showed little amino acid variations (Fig. 3 ). 


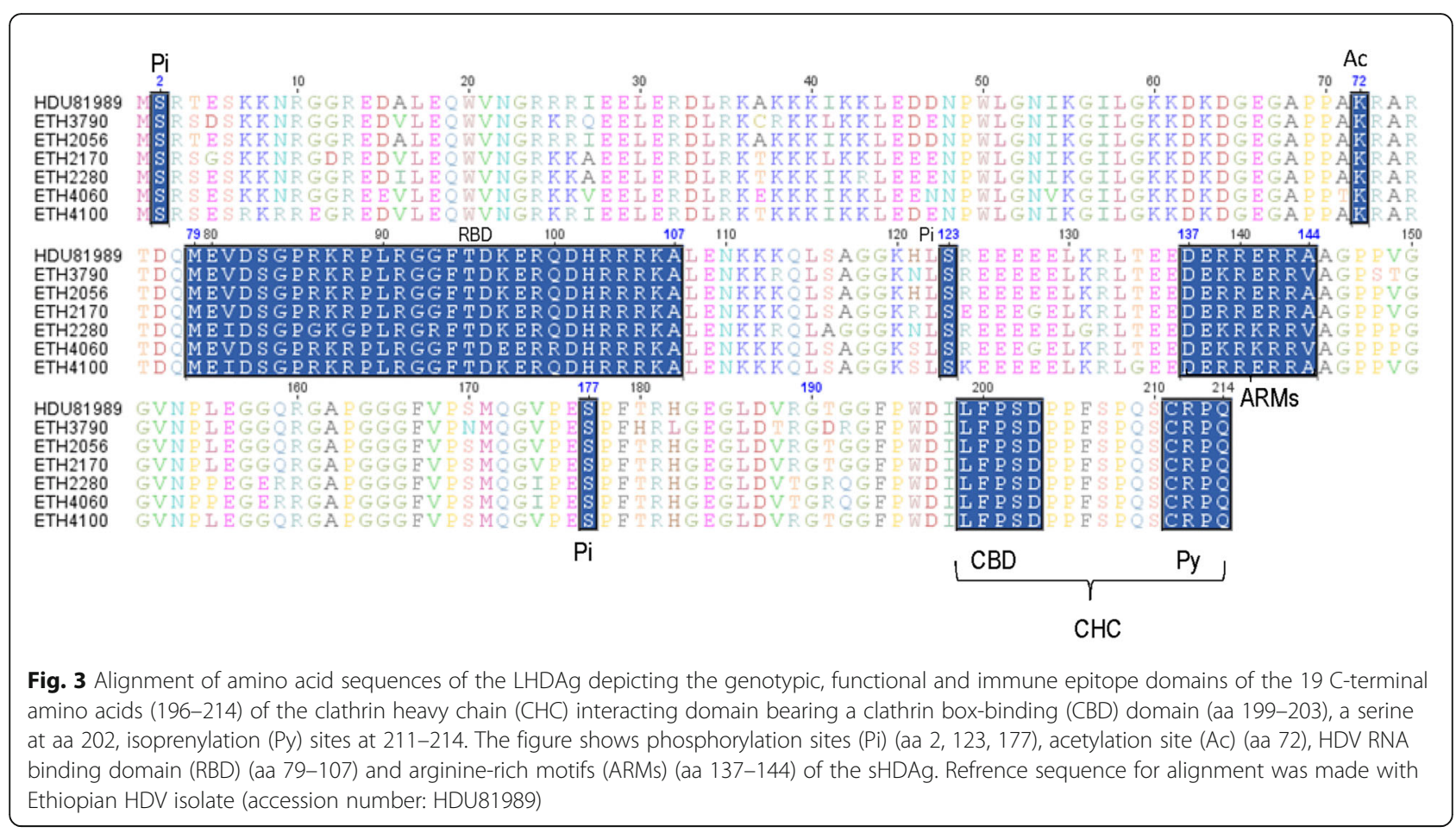

\section{Discussion}

HBV with a prevalence rate of $7.4-8.4 \%[14,16]$ is hyperendemic in Ethiopia but data on HDV seroepedimiology and molecular diversity are generally lacking. This study, to the best of our knowledge, is the first description of HDV molecular characterization from a large study population comprising blood donors, liver disease patients and HIV infected individuals. The seroprevalence and viral detection rate of HDV observed among HBsAg positives cases in this study were unexpectedly low when compared to previous reports from Ethiopia [17, 18], other African countries and global data $[7,10,19]$. Nevertheless, reports showed an increasing incidence of global HDV infection due to dynamic population movement particularly in HIVinfected patients from HBV hyperendemic area [10]. For instance, in a European cohort of HIV (EuroSIDA), $14.5 \%$ seroprevalence of HDV with $87 \%$ viral RNA detection rate was reported [7]. Moreover, in areas of west and east African regions where geographical proximity and healthcare access were more or less similar with the current study setting, up to $70 \%$ of viral detection rate was reported [19-21].

In this study, however, there appeared to be low prevalence and/or high HDV viral clearance which could contradict with the notation that during multiple viral infections such as other hepatitis viruses and HIV, HDV is usually dominant and inhibits the others viruses $[4,22]$. Especially, in immunodeficient patients, the HDAg was reported to stay longer because of the slow and weak immune response [23]. In contrast, more than $80 \%$ of anti-HDV antibody positive participants showed comparably high HBV DNA levels relative to reports elsewhere infected with HDV-1 genotype [4]. As the result, in particular HBV-HIV co-infected study population of the current study, it was necessary to examine the impact of more than a decade HIV ART exposure against $\mathrm{HBV}$ and consequent complex drug resistance and concomitant HBsAg variability profiles [14] on anti-HDV positivity and viremia rate. In this case, the variability of the surface protein might affect the degree of the antigenicity which potentially plays down HDV assembly and infectivity. It is known that certain amino acid sequences in the $\mathrm{C}$-terminal domain of the surface protein are essential for the assembly of HDV particles [24]. In the current HIV infected study subjects, the prevalence of YM(I)DD motif HBV-drug resistant mutations i.e. rtM204V/I accounted for $29.3 \%$ and 15.5\% rtL173V with concomitant immune escape HBsAg mutants of $13.8 \%$ rtM204V/sI195M, 3.4\%rtM204I/ sW196" and 10.3\% rtV173L/rtL180M/rtM204V + sE164D /sI195M [14]. Except one case (PCR negative for HDV), none of the HBV-HIV co-infected patients who were characterized by these concomitant drug and surface gene mutants were positive for anti-HDV antibody. The absence of anti-HDV positivity in this group, therefore, supports the above speculation and previous findings [7, 24, 25], although the HDV seroprevalence data of the current study did not consider HBsAg negatives due occult HBV infections and /or anti-HBc positive 


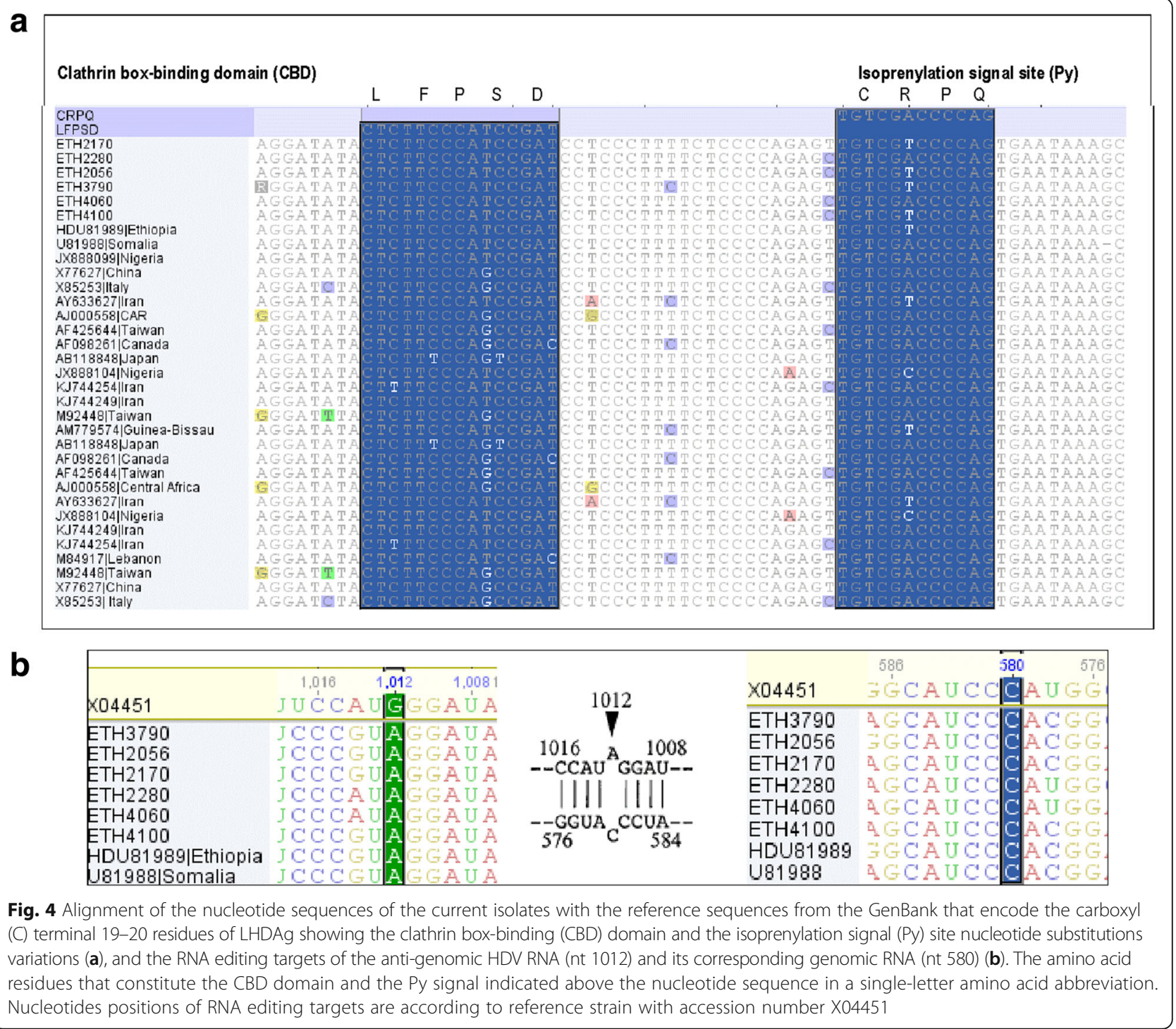

cases. For instance, the immune escape HBsAg mutations such sQ164A and sE164D with concomitant rtV173L were reported to reduce the antigenicity of HDV particles [25]. Moreover, the HBV drug resistant mutations (rtM204V/I) are associated with changes in the overlapping envelope gene products, in particular, the gene encoding small envelope protein (s) at sI195M or sW196L/S/* [24] which in turn can impair HDV replication by compromising viral particles assembly and secretion $[7,24]$.

The blood donors and CLD patients of the current study were characterized by relatively less frequency of immune escape HBsAg mutations than their HIV counterparts (but higher than similar reports elsewhere) [14] may also partly explains the unexpectedly low rate of HDV viral detection. A more limited production of HBsAg by HBV genotype A than D [7] coupled with the above scenario might contribute to explain this observation since genotype $\mathrm{A}$ is the predominant isolate in Ethiopia [26]. Moreover, except one case, three of the strains in the current study were isolated from patients with HBV genotype A unlike previous studies that reported HDV-1 was mostly associated with HBV genotype D $[4,27]$. However, in half of the HDV-1 isolates sequenced, HBV virus was suppressed to undetectable levels and thus co-genotyping of HBV was not possible and made the above explanation partial.

In this study, the phylogenetic analysis showed that all the six isolates were HDV-1 clustered into two monophyletic clusters and one of the clusters showed high relation to a previously described single strain from an Ethiopian patient isolated in Sweden [28]. Interestingly, this apparent segregation and independent clustering was reproducible when both the full genome and non- 
coding partial genome region sequences (368 nt) were considered for the phylogenetic analysis. Although HDV genotyping studies so far reported are very few $[9,13]$, analysis of HDV sequences from African countries showed the highest diversity of genotypes 1 and 5-8 $[29,30]$. However, complete presence of HDV-1 from the current and previous studies in Ethiopia and neighboring Somalia [28] suggest that HDV-1 was a predominant clade occurring in Eastern African regions although majority of east African countries still uncovered.

The clade homogeneity observed in the phylogenetic analysis was also reflected from analysis of amino acid residues and nucleotide sequences of those important structural and functional domains of the last $19 \mathrm{C}$ terminal of LHDAg [2, 31, 32]. Among the essential sequences for HDV replication and maturation, the sequence encoding the C-terminal peptide of LHDAg of genotypes HDV1-8 are with the highest diversity compared to other regions of protein-coding sequences [32]. HDV-1 in which the current strains belongs was characterized by ${ }^{196}$ WDILFPSDPPFSPQS-CRPQ ${ }^{214}$ amino acid sequences of the 19-C terminal protein $\mathrm{CHC}$ domain [32] with CBD amino acid sequence of 'LFP (A, S, V) D' located at amino acids 199-203 [2]. With no significant nucleotide substitution variations, all the current isolates together with previously identified Ethiopian strain [28] showed the ${ }^{196}$ WDILFPSDPPFSPQS-CRPQ ${ }^{214}$ amino acid sequences with the CBD sequences of ${ }^{199}$ LFPSD $^{203}$. Moreover, at aa position 202, they also exhibited a serine in contrast to Eurasian strains (which have an alanine residue) [4]. At the C-terminus of LHDAg which acts as a signal of isoprenylation for viron assembly and secretion are also characterized by amino acid sequence variations of ${ }^{211} \mathrm{CRPQ}^{214},{ }^{211} \mathrm{CTPQ}^{214}$, and ${ }^{211} \mathrm{CTQQ}^{214}$ in various HDV genotypes. Similarly, the HDV-1 of the current study showed ${ }^{211} \mathrm{CRPQ}^{214}$ with four out of the six isolates showing a nucleotide change from $\mathrm{A}$ to $\mathrm{T}$ at the Py signal site but the CRPQ amino acid sequences were unaffected. This further proved absence of a failure in viron assembly and secretion due mutations of the Py signal of LHDAg [2]. The CHC domain sequences of the current study also showed absolute conservation at nucleotides level when compared with nucleotide sequences of the nearest HDV-1 strains retrieved from GenBank on the basis of the Basic Local Alignment Search Tool. Absence of significant amino acid and nucleotide sequences variation in the $19 \mathrm{C}$-terminal LHDAg showed HDV viral assembly and infectivity were unaffected and had a less likely association with the low rate of viral detection in this study. Moreover, the nucleotide constitutes of the RNA editing targets of the anti-genomic HDV RNA at nt 1012 and its corresponding genome site at nt 580 showed a known of tryptophan codon (TGG) which is required for efficient RNA editing and HDV replication [33, 34] instead of the amber stop codon (TAG).

The sHDAg amino acid sequences which have structural and functional roles were also compared among the six sequences. The amino acid sequence analysis of the post-translational phosphorylation site in the sHDAg protein showed serine at positions 2, 123 and 177 which is responsible for genomic HDV RNA synthesis (HDV antigenome RNA replication) [35] by interacting with cellular RNA polymeraseII [36]. Moreover, the acetylation site aa 72 showed a lysine residue in this study in which its substitution by an alanine re-localized the mutant SHDAg into the cytoplasm and associated with the diminished viral RNA accumulation and earlier LHDAg appearance [37, 38]. In contrast, although variation of amino acid sequence requirement for the RNA binding function may not be that much strict [28], three of the six isolates showed variations only on two amino acids positions (V81I and R88G).

\section{Conclusions}

Exclusion of anti-HDV seroprevalence report from HBsAg negatives as the result of occult HBV infections and /or anti-HBc positive cases was the limitation of the current study. Despite a large study population screened in this study and hyperendemicity and genetic heterogeneity of HBV infection in Ethiopia, anti-HDV seroprevalence and HDV viremia rates were relatively low and even absent in those HBV-HIV co-infected with YM(I)DD motif drug selected HBsAg mutant variants. Thus, the potential role of HBV drug resistance associated polymerase mutations and concomitant surface protein variability on HDV viral assembly and infectivity needs further investigation. In contrast, the phylogenetic analysis showed the circulation of HDV-1 with the highest clade homogeneity and conservation of nucleotides and amino acid sequences on important structural and functional domains of HDV. This study provides new insights into the genetic diversity and molecular epidemiology of HDV-1 in Ethiopia where access to healthcare for viral hepatitis is totally absent.

\section{Abbreviations \\ 3TC: Lamivudine; aa: Amino acids; Ac: Acetylation sites; ARMs: Arginine-rich motifs; CBD: Clathrin box-binding domain; CHC: Clathrin heavy chain; \\ CLD: Chronic liver disease; HBsAg: Hepatitis B surface antigen; HBV: Hepatitis B virus; HCC: Hepatocellular carcinoma; HDAg: Hepatitis delta antigens; HDV- \\ 1: HDV genotype 1; HIV: Human immunodeficiency virus; IgG-Ab: Anti-HDV antibody; LC: Liver cirrhosis; PCR: Polymerase chain reaction; \\ Pi: Phosphorylation site; Py: Isoprenylation signal site; RT: Reverse \\ transcription; YM(I)DD: Tyrosine-methionine/isoleucine-aspartate-aspartate}

\section{Acknowledgements}

We are deeply grateful to Sandra Bergs, from Institute of Virology, Medical Faculty, Leipzig University, for her excellent technical assistance and consultation.

Funding

Received no specific grant to conduct the study. 


\section{Availability of data and materials}

The nucleotides sequence data used in this study analysis is submitted to GenBank.

\section{Authors' Contributions}

Conceived and designed the experiments: YB, MM, UGL. Performed the experiments and drafted the manuscript: YB. All authors read and approved the final version of the manuscript.

\section{Ethics approval and consent to participate}

Ethical approval was granted from the Institutional Ethical Review Board of University of Gondar (Ref. No: RCS/N/P/05/372/2013) and permission was obtained from the University of Gondar teaching Hospital, Debretabor Hospital, and Gondar Poly Health Centre, Ethiopia (Ref. No: CMHS/08/30/ 2013). Informed consent from each study participant was also obtained before data collection.

\section{Consent for publication}

Not applicable.

\section{Competing interests}

The authors declare that they have no competing interests.

\section{Publisher's Note}

Springer Nature remains neutral with regard to jurisdictional claims in published maps and institutional affiliations.

Received: 12 January 2017 Accepted: 5 September 2017 Published online: 12 September 2017

\section{References}

1. Sureau C, Negro F. The hepatitis delta virus: Replication and pathogenesis. J Hepatol. 2016;64(1 Suppl):S102-16.

2. Wang $Y$, Huang $C$, Chao M, Lo SJ. The C-terminal sequence of the large hepatitis delta antigen is variable but retains the ability to bind clathrin. Virol J. 2009:6:31.

3. Huang C. Hepatitis D virus infection, replication and cross-talk with the hepatitis B virus. WJG. 2014;20:14589.

4. Le Gal F, Badur S, Hawajri NA, Akyuz F, Kaymakoglu S, Brichler S, et al. Current hepatitis delta virus type 1 (HDV1) infections in central and eastern Turkey indicate a wide genetic diversity that is probably linked to different HDV1 origins. Arch Virol. 2012;157:647-59.

5. Wedemeyer $\mathrm{H}$, Heidrich B, Manns MP. Hepatitis D virus infection-Not a vanishing disease in Europe! Hepatology. 2007;45:1331-2.

6. Sy BT, Nguyen HM, Toan NL, Song LH, Tong HV, Wolboldt C, et al. Identification of a natural intergenotypic recombinant hepatitis delta virus genotype 1 and 2 in Vietnamese HBsAg-positive patients. J Viral Hepat. 2015;22:55-63.

7. Soriano V, Grint D, d'Arminio Monforte A, Horban A, Leen C, Poveda E, et al. Hepatitis delta in HIV-infected individuals in Europe. AIDS. 2011;25:1987-92.

8. Çelik I, Karataylı E, Çevik E, Gökçe Kabakçı S, Karataylı SC, Dinç B, et al. Complete genome sequences and phylogenetic analysis of hepatitis delta viruses isolated from nine Turkish patients. Arch Virol. 2011;156:2215-20.

9. Le Gal F, Gault E, Ripault M, Serpaggi J, Trinchet J, Gordien E, et al. Eighth major clade for hepatitis delta virus. Emerg Infect Dis. 2006;12:1447-50.

10. Hung C, Wu S, Lin P, Sheng W, Yang Z, Sun H, et al. Increasing incidence of recent hepatitis $D$ virus infection in HIV-infected patients in an area hyperendemic for hepatitis B virus infection. Clin Infect Dis. 2014;58:1625-33.

11. Hønge BL, Jespersen S, Medina C, DdS T, da Silva ZJ, Lewin S, et al. Hepatitis $B$ and Delta virus are prevalent but often subclinical co-infections among HIV infected patients in Guinea-Bissau, West Africa: a cross-sectional study. PLoS One. 2014;9:e99971.

12. Mendes-Correa MC, Gomes-Gouvêa MS, Alvarado-Mora MV, Da Silva MH, Lázari C, Cavalcanti NCS, et al. Hepatitis delta in HIV/HBV co-infected patients in Brazil: is it important? Int J Infect Dis. 2011;15:e828-32.

13. Andernach IE, Leiss LV, Tarnagda ZS, Tahita MC, Otegbayo JA, Forbi JC, et al Characterization of hepatitis delta virus in sub-Saharan Africa. J Clin Microbiol. 2014;52:1629-36.

14. Belyhun Y, Maier M, Liebert UG. HIV therapy with unknown HBV status is responsible for higher rate of HBV genome variability in Ethiopia. Antivir Ther. 2017;22(2):97-11.
15. Hofmann J, Frenzel K, Minh BQ, von Haeseler A, Edelmann A, Ross SR, Berg $T$, et al. Quantitative detection and typing of hepatitis $D$ virus in human serum by real-time polymerase chain reaction and melting curve analysis. Diagn Microbiol Infect Dis. 2010;67:172-9.

16. Belyhun Y, Maier M, Mulu A, Diro E, Liebert UG. Hepatitis viruses in Ethiopia: a systematic review and meta-analysis. BMC Infect Dis. 2016;16(1):761.

17. Tsega E, Mengesha B, Hansson BG, Lindberg J, Nordenfelt E. Hepatitis A, B, and delta infection in Ethiopia: a serologic survey with demographic data. Am J Epidemiol. 1986;123:344-51.

18. Rapicetta M, Hailu K, Ponzetto A, Hele C, Morace G, Bekura D, et al. Delta hepatitis virus infection in Ethiopia. Eur J Epidemiol. 1988:4:185-8.

19. Aghasadeghi MR, Mohraz M, Bahramali G, Aghakhani A, Banifazl M,

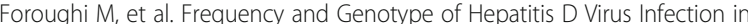
Patients Infected with HIV and Those Undergoing Hemodialysis. Hepat Mon. 2013;13:e7481.

20. Makuwa M, Mintsa-Ndong A, Souquiere S, Nkoghe D, Leroy EM, Kazanji M. Prevalence and Molecular Diversity of Hepatitis B Virus and Hepatitis Delta Virus in Urban and Rural Populations in Northern Gabon in Central Africa. J Clin Microbiol. 2009;47:2265-8.

21. de PM, Ceriani C, Cerulli T, Cagnin D, Cavallari S, Ndayaké J, et al. Prevalence of HBV, HDV, HCV, and HIV infection during pregnancy in northern Benin. J Med Virol. 2014:86:1281-7.

22. Pollicino T, Raffa G, Santantonio T, Gaeta GB, lannello G, Alibrandi A, et al. Replicative and Transcriptional Activities of Hepatitis B Virus in Patients Coinfected with Hepatitis B and Hepatitis Delta Viruses. J Virol. 2010;85:432-9.

23. Pascarella S, Negro F. Hepatitis D virus: An update. Liver Int. 2011;31:7-21.

24. Vietheer PTK, Netter HJ, Sozzi T, Bartholomeusz A. Failure of the LamivudineResistant rtM204l Hepatitis B Virus Mutants To Efficiently Support Hepatitis Delta Virus Secretion. J Virol. 2005;79:6570-3.

25. Salisse J, Sureau C. A Function Essential to Viral Entry Underlies the Hepatitis B Virus "a" Determinant. J Virol. 2009;83:9321-8.

26. Hundie GB, Raj VS, Michael DG, Pas SD, Osterhaus ADME, Koopmans MP, et al. Molecular epidemiology and genetic diversity of hepatitis $B$ virus in Ethiopia. J Med Virol. 2016:88:1035-43.

27. Saudy N, Sugauchi F, Tanaka Y, Suzuki S, Aal AA, Zaid MA, et al. Genotypes and phylogenetic characterization of hepatitis $B$ and delta viruses in Egypt. J Med Virol. 2003;70:529-36.

28. Zhang $Y Y$, Tsega $E$, Hansson BG. Phylogenetic analysis of hepatitis D viruses indicating a new genotype I subgroup among African isolates. J Clin Microbiol. 1996:34:3023-30.

29. Radjef N, Gordien E, Ivaniushina V, Gault E, Anais P, Drugan T, et al. Molecular phylogenetic analyses indicate a wide and ancient radiation of African hepatitis delta virus, suggesting a delta virus genus of at least seven major clades. J Virol. 2004;78:2537-44.

30. Deny P. Hepatitis delta virus genetic variability: from genotypes I, II, III to eight major clades? Curr Top Microbiol Immunol. 2006;307:151-71.

31. Huang C, Chang SC, Yu I, Tsay Y, Chang M. Large hepatitis delta antigen is a novel clathrin adaptor-like protein. J Virol. 2007:81:5985-94.

32. Huang C, Lo SJ. Evolution and Diversity of the Human Hepatitis D Virus Genome. Adv Bioinforma. 2010;2010:1-9.

33. Ivaniushina V, Radjef N, Alexeeva M, Gault E, Semenov S, Salhi M, et al. Hepatitis delta virus genotypes I and II cocirculate in an endemic area of Yakutia. Russia J Gen Virol. 2001;82:2709-18.

34. Watanabe H. Chronic hepatitis delta virus infection with genotype llb variant is correlated with progressive liver disease. J Gen Virol. 2003;84:3275-89.

35. Yeh T, Lee YW. Assembly of Hepatitis Delta Virus Particles: Package of Multimeric Hepatitis Delta Virus Genomic RNA and Role of Phosphorylation. Virology. 1998:249:12-20.

36. Hong S, Chen P. Phosphorylation of Serine 177 of the Small Hepatitis Delta Antigen Regulates Viral Antigenomic RNA Replication by Interacting with the Processive RNA Polymerase II. J Virol. 2010;84:1430-8.

37. Abbas Z. Life cycle and pathogenesis of hepatitis D virus: A review. WJH. 2013;5:666.

38. Mu J, Tsay Y, Juan L, Fu T, Huang W, Chen D, et al. The small delta antigen of hepatitis delta virus is an acetylated protein and acetylation of lysine 72 may influence its cellular localization and viral RNA synthesis. Virology. 2004:319:60-70 